\title{
LA SUBJETIVIDAD Y LA INTERSUBJETIVIDAD: DOS VECTORES EN LA EVALUACIÓN COMO FENÓMENO ANTROPOLÓGICO'
}

\section{SUBJECTIVITY AND INTERSUBJECTIVITY: TWO VECTORS IN EVALUATION AS AN ANTHROPOLOGICAL PHENOMENON}

\author{
Jorge H. Betancourt Cadavid ${ }^{2}$ \\ Johann Enrique Perez ${ }^{3}$ \\ Universidad San Buenaventura
}

\section{RESUMEN}

El presente artículo asume la subjetividad e intersubjetividad como nociones fenomenológicas al interior de la evaluación

$\overline{1}$ Artículo de investigación resultado de la tesis de Doctorado en Ciencias de la educación en la Universidad de San Buenaventura de Medellín, en la línea de Formación y Subjetividad. Dicha indagación tiene por título La constitución del sujeto en la evaluación educativa, una aproximación desde la antropología fenomenológica.

2 Doctor en Filosofía (PhD), especialista en docencia universitaria y licenciado en educación. Doctor Honoris Causa por la Organización Continental de Excelencia Educativa - ORCODEE (2019). Pasante de posdoctorado en Educação del Instituto Federal Sul-Rio-Grandense de Brasil. Docente investigador de la maestría y el doctorado en ciencias de la educación de la Universidad de San Buenaventura, y de la facultad ciencias de la educación en la Corporación Universitaria Americana, ambas instituciones en Medellín (Colombia). Contacto: jorge.betancourt@usbmed.edu.co ORCID: 0000-0003-3286-870

3 Candidato a Doctor en Ciencias de la Educación de la Universidad San Buenaventura. Magíster en Procesos de enseñanza y aprendizaje de segundas lenguas, especialista en enseñanza del inglés y licenciado en lenguas modernas español-inglés. Docente vinculado a la planta de cargos de la Secretaría de Educación departamental de Antioquia. Investigador adscrito al Grupo Interdisciplinario de Estudios Pedagógicos - GIDEP. Contacto: perezjohann@hotmail. com ORCID: 0000-0003-0398-5426 en la educación para interpretar la vida humana inherente a ella. Lo hace desde estos dos vectores, fundamentando una búsqueda de elementos que, desde la antropología fenomenológica consolidan dentro del campo de la pedagogía una reflexión filosófica en torno a la constitución del sujeto y los otros de su entorno en la evaluación, describiendo la correlación entre las operaciones o efectuaciones constituyentes del mundo y lo efectuado en estas; se trata de la alusión a un Yo que cobra su sentido de las efectuaciones de la conciencia, pero que no es un Yo solo en el mundo, como una mónada incomunicable, sino en presencia e interacción con otro u otros con los que comparte un mundo, como condición de posibilidad de que un Ego pueda interactuar con el Otro para concretar su humanidad. Esta búsqueda se describe en torno a cuatro apartados que constituyen el cuerpo del articulo y cada uno argumenta la premisa de que la evaluación es una experiencia humana, 
por ende, un fenómeno antropológico que supera la reiterativa y afincada descripción de la evaluación como dispositivo.

\section{PALABRAS CLAVE}

antropología fenomenológica, subjetividad, intersubjetividad, evaluación.

\section{ABSTRACT}

This article assumes subjectivity and intersubjectivity as phenomenological notions proper to evaluation, and necessary to interpret human life inherent to it. It does so from these two vectors, based on a search for elements that, from phenomenological anthropology, consolidate within the field of pedagogy a philosophical reflection on the constitution of the subject and the others in their environment in the evaluation, describing the correlation between the operations or activities that constitute the world and what has been done in them. It is about the allusion to an " $l$ " that receives its meaning from the effects of consciousness, but that is not an I alone in the world, like an incommunicable monad, but in the presence and interaction with another or others with whom it shares a world, as a condition of possibility that an Ego can interact with the Other to specify its humanity. This search is described around four sections that constitute the body of the article and each one argues the premise that evaluation is a human experience, therefore, an anthropological phenomenon that goes beyond the reiterative and established description of evaluation as a device.

\section{KEYWORDS}

Phenomenological anthropology, subjectivity, intersubjectivity, evaluation.

\section{INTRODUCCIÓN}

Si puedo tocar, experimentar y sentir el mundo, es sólo porque éste "yo", antes de que pudiera nominarse lo agarraron, lo sintieron, se dirigieron a él y lo animaron.

(Butler, 2016, p. 23)

Se concibe la evaluación como una realidad en cuyo interior fluctúa la subjetividad como territorio existencial del sujeto, la cual, en su conjunto de condiciones sociales e históricas, cargadas de construcciones simbólicas e imaginarias, dinamiza para el sujeto la producción de sentido y el actuar sobre la realidad, además de sus definiciones identitarias. Son estas reflexiones que ponen de manifiesto de los autores la urgente necesidad de una comprensión en torno a la evaluación en los procesos de educación y la intención de la formación humana en tiempos como estos, asuntos que no pueden seguir centrados de manera exclusiva desde el ambiente escolar en la escuela (BetancourtCadavid, 2015).

Esta reflexión se funda desde el orden epistemológico, en la construcción de una propuesta pedagógica no paradigmática. Es la ruptura con la pregunta en torno al ordenamiento de la cotidianidad, y el quiebre con las respuestas que ofrecen a los seres humanos las pedagogías convencionales, ya el estudio sobre la formación se ha habituado con el paso de los siglos. (Betancourt-Cadavid, 2012, p. 44).

Aquí se sigue uno de tres movimientos de análisis que constituyó la perspectiva metodológica del presente estudio y que orientó la indagación de tipo teórica, que encarnó una reflexión pedagógica desde el terreno de enunciación de la antropología fenomenológica. Por lo tanto, se propendió por establecer cómo la evaluacióncomo dispositivo y fenómeno antropológico permite o no el desenvolvimiento del sujeto al que se alude en la Antropología Fenomenológica. Se partió desde la premisa encontrada y justificada 
en el segundo capítulo de este estudio, en el sentido de que no se puede pensar la evaluación antes que la experiencia evaluativa, toda vez que la evaluación no precede al sujeto que la experimenta, es la condición afincada en la noción de sujeto, de vida humana, como central para la validez del mundo y la constitución de su sentido, en el que la evaluación deviene en experiencia humana.

Ahora bien, el concepto de subjetividad con que opera Edmund Husserl alude a un sujeto libre $\mathrm{y}$ autorresponsable por un lado, y al concepto de subjetividad trascendental, por otro lado, lo cual indica la correlación entre las operaciones o efectuaciones constituyentes del mundo y lo efectuado en estas, alude a un yo que cobra su sentido de las efectuaciones de la conciencia, pero que no es un yo solo en el mundo, como una mónada incomunicable, sino en presencia e interacción con otro u otros con los que comparte un mundo, como condición de posibilidad de que un ego pueda interactuar con ese otro y concretice su humanidad.

Empero, desde el plano de la percepción, yo y cuerpo no pueden confundirse, bajo el esquema dual de lo humano, cada sujeto posee un cuerpo que no viene a estar determinado unívocamente por su estructura biofísica, sino desde sus dos planos corpóreos del yo vivo y el yo vivido que le habitan a esa estructura que hace su entrada en el mundo perceptible del otro, donde ese yo vivido constituiría la zona más íntima del sujeto por ser su yo inmaterial.

Son dos problemas a los que Husserl accede de manera explicativa en su obra como parificación y cuyo núcleo es la apresentación, concepto fundante en la intersubjetividad y al que remite Husserl para explicitar que la forma originaria con que opera la conciencia está determinada en el cómo esta unifica diversas vivencias en la experiencia de un objeto, delimitando que el modo de darse los objetos en la conciencia no son una serie inconexa de representaciones o apariciones que, por el contrario, aparecen en una unidad sintética de vivencias desde la cual se constituye el sentido en la conciencia, desde donde se elabora sintéticamente la noción de ese objeto para el yo que le constituye y cuya efectuación genera una afectación que cobra sentido constitutivo en ese yo, "la constitución del objeto de que se trate en cada caso se produce mediante la síntesis en una unidad de los múltiples y multiformes modos en que dicho objeto aparece en la vida fluyente y continua de la conciencia" (Álvarez, 2011, p. 123).

Desde estos dos problemas que aborda y explica Husserl a lo largo de su obra y que edifican su método de la fenomenología genética, en síntesis, definido como reconstrucción de la génesis del sentido a partir de la experiencia originaria dada en el mundo de la vida (Vargas Bejarano, 1993, p. 105), se logra determinar que lo que conocemos como realidad no es algo acabado que se nos presenta y vivimos de manera ingenua, sino que esas vivencias son trascendidas desde un yo que en sus intencionalidades y operación de conciencia les constituye y es constituido por el sentido que las mismas se efectúan en él y que, por tanto, al ser la evaluación educativa una experiencia humana, ha de estar determinada por esa bilateralidad de dicha constitución en operación sintética e intersubjetiva.

\subsection{El problema de la apresentación en el concepto de evaluación}

Al concebir la evaluación como fenómeno antropológico, en cuyas bases está la posibilidad de esclarecer si la noción del sujeto que se evalúa hace justicia al sujeto humano, ese sujeto es vida, no cosa, donde la evaluación, debe ser tomada como una práctica dirigida a un algo que no es algo, sino un quién, lo cual devela que las actividades judicativas en ella no deben ser arbitrarias en términos de la experiencia, por lo que exigen ser pasadas por el cedazo del análisis de sus estructuras esenciales, para el caso de la 
evaluación como fenómeno antropológico exige ser analizada desde las estructuras del yo y el otro como condición y objeto de la experiencia.

Esta concepción de la que se habla para aclarar el concepto de sujeto, se mueve inmerso en la intención de proporcionar elementos a los problemas que le son pertinentes al campo disciplinar y profesional de la pedagogía. La idea es, en corcondancia con la propuesta de Bourdieu y Wacquant (2005), sabiendo que dentro de este campo "Estas posiciones se definen objetivamente en su existencia y en las determinaciones que imponen a sus ocupantes, ya sean agentes o instituciones" (Runge Peña, Hincapié, Muñoz y Ospina, 2018, p. 236). Por eso es que el artículo participa como resultado de investigación de un régimen particular de relaciones objetivas dadas entre los agentes interesados disciplinarmente en este lugar como espacio de unos discursos en torno a la formación y la educación.

Así las cosas, permítase el abordaje de la obra de Husserl en este campo para cavilar sobre la actividad de conciencia que consiste en la intencionalidad y no en las formas con que ella capta el objeto, por lo tanto, el objeto se revela referido al acto intencional del sujeto. Es la superación de la histórica dialéctica entre sujeto- objeto, porque el énfasis no se queda en el plano de lo mundano sobre el objeto que co- existe al sujeto psicofísico que le percibe, el plano va más allá por la implicación interna en el sujeto que determina desde su conciencia y su intencionalidad el mundo mismo, un ego cuya experiencia no se ancla en lo referido a su experiencia del ser natural del mundo como un yo- mundano, sino que le exige como un yotrascendental que es condición de posibilidad del mundo mismo, en el que se encuentra el sentido a su radical experiencia del mundo.

En consecuencia, si bien el mundo me precede materialmente y el yo precede a su idea del mundo, es en ese yo donde las cosas pueden adquirir o no sentido en cuanto lo son para o de la conciencia, en tanto que el yo no sólo es constituido en el mundo, sino constituyente en él y para él, "Por su parte, Husserl rechaza que se pueda hablar con sentido de un dato que la conciencia haya de admitir como algo impuesto externamente a ella de modo absoluto" (Álvarez, 2011, p. 109). De igual manera Husserl rechaza la consideración sustancialista de ese yo, porque no es solo implicado, encerrado en sí mismo, sino en relación con el mundo, con lo cual se aparta Husserl del radical dualismo de las sustancias.

Sin embargo, y cómo ya se ha referido al inicio de este artículo, esta relación con el mundo no es una experiencia que se da como realidad impuesta de manera absoluta al yo que la experimenta, ni es ese yo solo en el mundo constituyéndola de sentido, porque coexiste con otros yoes que también son capaces de operar intencionalmente sobre ese mundo. Por lo tanto, es necesario tener presente que, en cuanto experiencia del campo perceptivo de la vida intencional de mi yo, entra a ser determinante en la instauración originaria de ese otro yo que coexiste conmigo en el mundo, donde mi yo también resulta determinante en su yo personal.

Remite, entonces, el concebir la evaluación como escenario donde el otro o los otros pueden $o$ no tener sentido y validez para el yo que se constituye en mí, en el ámbito de determinaciones y asimilaciones en el reconocimiento de un yo frente a otros, en correlación con un mundo de co- pertenencias y unidades de sentido vivenciales en las posibilidades que se despliegan y fluctúan en el trascender de la experiencia de un yo psicofísico que alcanza y dinamiza funciones esenciales en su vida de conciencia, donde como yo-ego no solo me experimento a mí, sino también al otro.

Por lo tanto, se edifica desde aquí la pregunta por el sujeto desde la fenomenología en Husserl, desde su doble perspectiva en el sentido 
de la vivencia intencional y la operatividad de conciencia en un yo- trascendente con inmanencia a ese sujeto. Desde esta doble perspectiva se halla al sujeto que es yo- ego que reflexiona desde sus condiciones y motivaciones, pero que también es objeto de esas reflexiones en su estrato constitutivo.

En la cuarta meditación de Meditaciones Cartesianas, Husserl describe un yo como el ego constituyéndose continuamente así mismo como existente, el ego se capta no meramente como vida que fluye sino también como yo, el que vive esto y aquello. Es un yo con capacidad de decisión valorativa y volitiva, que se transforma cuando cancela o abandona esas decisiones que le hacían ser cierto yo en medio de las habitualidades en las que se determinaba por cierta decisión, lo que le constituye en el fluir de las vivencias, a través de las cuales y mediante la modelización de sus determinaciones acredita una unidad de identidad un carácter que le es propio.

Esto es, yo como ego tengo un mundo circundante que de manera continua existe para mí. El conocimiento de los objetos existentes en este mundo para mí son algunos ya conocidos y otros de conocimiento anticipado. En la primera hay una actividad de mi yo como actividad sintética en la forma en la que se constituye para mí ese objeto cómo identidad de este desde sus múltiples propiedades y que instaura en mí una propiedad permanente de sus determinaciones, esa propiedad permanente es ahora una habitualidad en mi yo. Los de conocimiento anticipado son objetos que me son desconocidos, pero que mediante mis adquisiciones permanentes puedo constituir y anticipar la estructura formal de ese objeto que aún me es desconocido.

Conduce, entonces, a la pregunta acerca de cómo experimentamos al otro o lo otro, desde el punto de vista fenomenológico, cómo se constituye para sí y para mí en la experiencia humana de la evaluación, en términos de experiencia del conocer, más que de validación del conocimiento como frecuentemente se le asocia al término. El yo descrito por Husserl en sus meditaciones no es un yo vacío de identidad ni tampoco lo es el objeto o el otro, lo otro o a los otros al que en un acto de conocer y dotar de sentido ese yo se aproxima. Es justamente con cada acto de sentido que se irradia y se gana una propiedad nueva que deviene en constituyente tanto para el yo que percibe como para el objeto o el otro que es percibido, o sea que la donación de sentido no es absoluta sino constitutiva y sintética, lo que forja una serie de determinaciones habituales en la corriente y fluir de las vivencias.

En ella están presentes las dos formas universales de los principios universales de la génesis constitutiva de nosotros como sujetos en correlato con el mundo. La génesis activa, constituyente por medio de datos del yo como efectuaciones de la razón práctica, donde se constituyen unos objetos sobre la base de objetos ya dados en los modos de conciencia y se presentan a la conciencia como productos.

Por su parte, la génesis pasiva referencia como grado inferior una pasividad - predonante, lo que la vida se nos presenta como concluido, como mera existencia de algo en la síntesis de la experiencia pasiva. Como cosas u objetos pre-dados a las captaciones de las síntesis de las experiencias activas. El ego que medita al penetrar en el contenido intencional de los fenómenos de la experiencia puede encontrar referencias intencionales que conduzcan a la historiaconstitutiva o sintética de dicho fenómeno, es decir las formaciones subsecuentes a otras formaciones que le precedieron en su esencia.

Como instauración originaria de esa forma todo lo transcurrido en la conciencia del yo como hacer el mundo dado, a partir de sus vivencias, le constituyen y le determinan como yo que desde sí válida a un mundo con el que entra 
en correlato y que cobra significación objetiva sobre sus vivencias gracias a la reducción fenomenológica, latitud fenomenológica que lo aleja de experimentar el mundo como sólo externalidad de la actitud ingeniosa, que le constituye como unión trascendental que le dimensiona con sentido y validez del mundo en sí y que le constituyen en su subjetividad como efectuación de su ego trascendental, con respecto a los horizontes que determinan su ser y sus correspondientes tareas intencionales a partir de los cuales se constituye desde sí y para sí, que le permite validarse como ego para construir al otro o lo otro en la interrelación intersubjetiva de cómo el otro vale para mí desde las implicaciones que comparten sus estructuras sintéticas que lo definen como un $y o$, pero que desde su subjetividad y estructura egoíca lo determinan como un no - yo en mi yotrascendental.

Al respecto Husserl argumenta que experimentamos algo inicialmente desde la génesis pasiva, donde aprendemos a ver primeramente las cosas y que tal aprendizaje precede genéticamente a todas las demás operaciones intencionales de conciencia sobre lo que percibimos, a partir de los cuales se van generando formaciones subsecuentes $y$ sintéticas a constituir eso que percibimos en un qué o quién en nuestra conciencia y a partir del cual determinamos un conocimiento que no deviene en cerrado o en absoluto.

Yo como sujeto que experimenta algo percibo un objeto no en su totalidad porque en la experiencia de percibirlo sólo obtengo de él un registro en el cual están implicadas cualidades y características que no se me dan en la primera percepción pero que asocio o parifico a otras experiencias de otros objetos similares que haya percibido previamente y que en medio de esa parificación perceptiva inscriba desde mis intencionalidades de percepción unos registros aperceptivos porque no los veo en él pero me adelanto en lo preconcebido y doto a ese objeto de lo que creo hay en él desde esa intención de apercepción, como configuraciones que le agrego a ese algo que percibo desde mis habitualidades ya determinadas en mí y que me han constituido y con las que ahora constituyo eso otro o ese otro que percibo, que aparecen como datos previos formados en $\mathrm{mi}$ yo que actualizan mi percepción desde lo que apercibo y que han de actualizar mi motivación y acción de conocer en la medida en que esas apercepciones pasen de ser un predicado aún por conocer a acceder al conocimiento de lo no conocido y que ha de convertirse en algo ya conocido en mi experiencia de ese objeto.

Esta problemática de la apercepción deviene en la experiencia evaluativa como el argumento por el cual no se debe adjudicar valoraciones o juicios absolutos sobre un sujeto que en medio de evidenciar un aprendizaje realice una acción y sobre ella se establezca un valor de jerarquización que califica dicho aprendizaje, porque no se puede determinar como absoluto si un sujeto ha aprendido o no sobre algo mientras está constituyendo desde su síntesis pasiva el conocimiento sobre cierta realidad u objeto de conocimiento, porque se estaría juzgando o calificando desde una percepción a priori o desde apercepciones que no son concluyentes en ese yo que es evaluado.

\subsection{De la síntesis pasiva a la intersubjetividad}

Con el reconocimiento de las capas pasivas de los actos de conciencia, como experiencias previas y fundantes de las capas activas de un yo que constituye en su correlación con el mundo, se abre paso en el análisis genético la búsqueda del origen de que eso que se me presenta en la experiencia es gradualmente constituyente de una intencionalidad que es posibilidad de revelación y de constitución del mundo en la conciencia de ese yo que vive y experimenta el mundo.

Se trata del conocimiento que emerge de ese vínculo constitutivo de la conciencia del yo- 
ego y el mundo, pero que no está radicalizado en los actos del yo para conocer, porque si bien Husserl ha explicado en la cuarta de sus meditaciones porqué la subjetividad es condición de posibilidad de toda objetividad, los actos de sentido y conciencia en esa correlación con el mundo poseen estratos pasivos que delimitan la característica constituyente de la objetividad sobre el sujeto que le percibe, siente, experimenta, conoce y finalmente constituye desde el sentido que logra su conciencia. Esto es, no reflexiono o conozco de manera arbitraria sin ser constituido en mi subjetividad por la objetividad de lo que percibo y experimento desde el fluir de la experiencia en una síntesis que se constituye desde sus capas de agencia pasiva hasta las dimensiones activas de la conciencia.

Es un descentramiento entre las concepciones de un sujeto activo y de un yo que no es sólo conciencia, porque en el influjo de la experiencia hay inherentes unas dimensiones pasivas que, al igual que las activas, son constitutivas desde dentro y fuera del sujeto que experimenta. Es un proceso mediante el cual el sujeto en sus vivencias da progresivamente sentido a los objetos, tal sentido no surge de la nada ni del simple y radical acto de observar y con ello conocer, sino que, desde la síntesis de dimensión pasiva, estos últimos se manifiestan en un horizonte externo de posibilidad: el mundo (Vargas Bejarano, 1993).

En su libro La fundamentación pasiva de la experiencia. Un estudio sobre la fenomenología de Edmund Husserl, Andrés Osswald (2016) hace una delimitación detallada de la pasividad como campo temático en la obra de Husserl y de sus alcances para teorizar sobre el sujeto. Su estudio descriptivo y analítico permite contraponer desde el concepto de pasividad la idea de una subjetividad centralizada en un yo provisto unívocamente con sus procesos activos. A partir de la descripción y análisis de lo que representa para el sujeto los estratos pasivos constitutivos para las dimensiones activas en su conciencia, Oswald explica cómo la noción de sujeto se amplía desde esas dos dimensiones constitutivas que describió Husserl en su obra y que, según dicho autor, el tópico de la pasividad recibió escasa atención en la primera recepción de la fenomenología husserliana.

En efecto, uno de los textos que más referencia Oswald en su libro para abordar el concepto de pasividad es el volumen XI de Husserliana: Analysen zur pasiven Synthesis. Aus Vorlesungs- und Forschungsmanuskrpten 1918-1926, editado por Margot Fleischer y publicado en 1966. Desde este y otras fuentes bibliográficas, este autor se permite un análisis consagrado a las estructuras de la pasividad, que permiten dar con los niveles primigenios del sujeto. De tal forma que las dimensiones de pasividad y actividad no son comprendidas como dimensiones excluyentes sino progresivas y de relación gradual y continua, que en doble vía determina la pre- delineación pasiva de los actos de un yo en correlación con el mundo y el fundamento yoico de la fenomenología como sujeto que es constituido y constituyente en dicha correlación.

Para Oswald el concepto de pasividad debe ser pensado en relación con la intencionalidad y establece que el alcance de esta última se redefine hacia niveles de la conciencia que no se identifican con un yo atento a lo que percibe y experimenta. Tal dimensión pasiva supone, entonces, un vínculo gradual entre el yo y la razón que hacen contiguos los estratos de la subjetividad, donde las nociones de parificación, apercepción, asociaciones descritas por Husserl en la cuarta y quinta meditación, aunadas al concepto de afección, son fundantes en la dimensión pasiva.

Este último, es descrito como el campo de la experiencia donde las unidades que sintetizan y se asocian entre sí mediante la síntesis pasiva se destacan de ejercer influencia sobre el yo y 
de atraer su atención de manera eventual. Es entendido, entonces, como el problema de dar cuenta del estímulo afectante de la asociación y síntesis de datos en una experiencia, con la posibilidad que el yo responda a ellas y empiece a generarse no sólo una atención subjetiva, sino a configurarse desde ella el sentido constitutivo.

Oswald analiza cómo la dimensión pasiva está en la base del desarrollo constitutivo del mundo, en la medida en que ella es responsable de desplegar todas las estructuras de la subjetividad y por ser el mundo su correlato, no solo se desarrolla la subjetividad, el mundo también. Tal gradualidad de desarrollo y despliegue implica una gradualidad de alcance y despliegue de la razón que desde el orden fenomenológico va desde el perfeccionamiento de lo instintivo al perfeccionamiento intencional de lo reflexivo y trascendental en la conciencia.

Desde el énfasis que Oswald hace en su estudio sobre el concepto de pasividad, permite redefinir el fundamento que comporta al sujeto como constituido y constituyente, no como un sujeto centrado en sus actos de conciencia sino desde la gradualidad que inscribe su correlación con el mundo que no inicia desde la acción sino desde la dimensión de la síntesis pasiva, lo cual inscribe el cómo se originan los procesos constitutivos de la subjetividad en la experiencia de lo humano y el alcance gradual de la razón, cuyo énfasis resulta fundamental para abordar la presencia del sujeto en la experiencia evaluativa como experiencia humana y no cosificada desde el orden de lo instrumental- regulativo.

Determina, por lo tanto, asumir el fenómeno de la experiencia evaluativa desde esa doble dimensión explicada por Husserl y analizada por Oswald sobre la base de la constitución de la subjetividad en sus dimensiones pasiva y activa, en esa dualidad en la que se inscribe una progresiva constitución desde las sedimentaciones instintivas hasta el ideal de un

acrecentamiento de la razón, entendida la vida instintiva no como un ejercicio ciego y mecánico o el ideal del alcance progresivo de la razón como una reificación absoluta que le llega de golpe a un sujeto.

En efecto, en su estudio Oswald describe que a través de ese proceso de desarrollo se constituye un sujeto concreto desde dos estratos que conforman la dimensión pasiva y que interviene en dicha constitución: El nivel de la vida instintiva y el de los hábitos adquiridos por el ejercicio de los instintos. Desde ahí describe que para que un sujeto se forme como persona debe suponer un desarrollo en una dirección ascendente de la etapa infantil hacia la adulta, pasando por tres niveles de constitución de las estructuras pasivas en la infancia:

El desarrollo infantil supone, al menos, tres niveles en la constitución de las estructuras pasivas de la subjetividad: la formación de la estructura temporal y asociativa, las estructuras que están en la base de la formación de los hábitos y la formación de los hábitos en sí mismos. Un tercer nivel, finalmente, residiría en volverse activo del yo sobre su vida pasiva. Se trata, naturalmente, de la formación del sujeto como persona propiamente dicha (Osswald, 2016, p. 218).

Ahora bien, Oswald advierte que tal despliegue monádico no está garantizado porque el desarrollo armónico de la monadología no depende de una unidad preestablecida, sino que, por el contrario, esta se constituye a partir de un proceso teleológico que le impulsa vinculado a la dimensión pasiva de la razón y a la dimensión activa que agencia las decisiones del sujeto que orienta sus acciones. Las dimensiones de vida yoica que tienen como correlato objetivo una cierta forma del mundo, constituyen un proceso con una direccionalidad abierta, por lo que Husserl concluye que el despliegue que se presupone no necesariamente haya de producirse y así, tal desvío o anquilosamiento 
del proceso o la razón siembra sus causas en las acciones de los hombres y no en la teleología que impulsa su desarrollo.

La aspiración racional- practica en virtud de la autodeterminación genérica de lo humano, está determinada por su hacer practico, valorativo y teórico en los aspectos de su vida, por lo que la racionalidad exigida a la condición humana tiene un carácter ideal; "por ello, la condición del hombre no se define en términos de una oposición estricta entre razón y sin razón sino que debe ser pensada, mejor, como la situación de quien habita el campo que reúne lo efectivo y lo posible, lo real y lo ideal y lo finito y lo infinito" (Osswald, 2016, p. 238).

Esto nos lleva a considerar aquellos hábitos y sedimentaciones pasivas de orden secundario que presuponen un acto y que tienen su institución originaria por asociaciones culturales o desde ciertas tradiciones, lo cual les devela como sedimentaciones o hábitos de carácter común, dejando a merced la correlación del sujeto con su mundo con la prescripción de dicho sentido y estructuras de sedimentación colectiva.

Es el paso de la síntesis pasiva al valor de lo intersubjetivo donde encuentra Husserl la tarea de discutir lo obvio, lo presupuesto y detenerse desde el nivel subjetivo a reflexionar y poner en suspensión fenomenológica lo que se me da, es la actitud que se reclama en oposición de habitar y vivir el mundo de manera ingenua y desprovista de la doble acción progresiva, vinculante y constituyente del tránsito de las dimensiones de lo pasivo a lo activo, entendiendo que la dimensión pasiva no constituye ya ese carácter de ingenuidad ante el mundo sino el suelo sintético para las acciones intencionales de la conciencia en un yo que es constituyente. Esto implica que como sujeto tiene la capacidad de frenar esa descarga de síntesis pasiva que le sobreviene por tendencias, creencias o tradición

y poner en cuestión tales presupuestos en el decurso de su tránsito y su posicionamiento hacia la dimensión activa.

\subsection{La subjetividad y la intersubjetividad desde el escenario de la antropología fenomenológica}

Desde la descripción husserliana de las dos dimensiones constitutivas, se concibe, entonces, que los otros, en cuanto otros, no existen realmente en mí, sino que de ellos tengo conciencia, configurándose en mí el sentido de alter- ego, los otros como objetos psicofísicos, pero que no como simple representaciones externas, sino que los otros son en el mundo desde sus yo- egos y que para mi yo-ego son "extraños" desde el término utilizado por Husserl en sus Meditaciones. Yo los asumo en mi experiencia como sujetos para ese mundo, el mismo mundo en el que yo experimento y en el cual ellos saben de mí como experiencia para ellos. Sin embargo, el sentido que ese mundo tiene para mí es un sentido desde mi vida intencional, pero el yo se encuentra a sí mismo como miembro de las exterioridades del mundo, la subjetividad que se despliega sobre la relación de las intersubjetividades. Es el yo que constituye y se constituye a sí mismo en su correlato con el mundo que existe para él como fenómeno, así lo plantea Husserl en el $§ 45$ de Meditaciones Cartesianas:

He llegado a percatarme de mí como ego trascendental que en su vida constitutiva constituye todo lo que en cada caso es para mí objetivo -el yo de todas las constituciones, que existe en sus vivencias actuales y potenciales, y en sus habitualidades personales (ichliche), en las cuales constituye todo lo objetivo y también se constituye a sí mismo como ego idéntico-. ( $p$. 133)

Así como yo me capto para mí en un yo- dado, originalmente intuible y con un horizonte abierto e infinito de propiedades interiores, en su gran mayoría aún no descubiertas, cobra sentido al 
captar al otro desde la actitud fenomenológica que ese otro es un yo- dado con infinitud de propiedades en su interior y con un horizonte abierto desde su "yo-soy", con una síntesis continua y unitaria de la originaria experiencia de sí mismo, que lo identifica y determina como yo en un mundo de correlación con otros yoes.

El otro, al igual que yo, tiene una naturaleza y corporalidad orgánica que se constituye como una unidad objetiva espacial, pero también trascendente, frente a la corriente vivencial en una experiencia, donde esta experiencia es mi vida, es el yo viviendo la experiencia, y no nada más que una unidad sintética inseparable de esa vida, porque al vivirlo ya le es esencialmente propio, le pertenece a su fluir de conciencia, a su morada, como concreción de su yo. Esta comunización de mónadas que se constituyen en su correlato con el mundo constituyen intersubjetivamente el mundo objetivo, donde una subjetividad para ese mundo, también lo es para las otras subjetividades como sistemas de síntesis constitutivas mutuamente correspondientes y concordantes.

Esta experiencia motiva una coexistencia que va de lo percibido a las apresentaciones, entendidas estas últimas como aquello que no se percibe pero que desde la vida intencional de mi yo-ego anticipa el horizonte lo que se percibe, por remisión intencional a una_instauración originaria de ese "algo" o "quien" objeto o sujeto de similar sentido, como suerte de transferencia analogizante de un sentido objetivo en una experiencia previa de sentido similar, lo que Husserl determina como predonación de sentido. Sin embargo, desde el punto de vista fenomenológico, el otro, como concreción de este otro yo, se presenta como una modificación de mi yo objetivado, se constituye en la mónada de mi yo, aprensivamente otra mónada, como correlato de la presencia del otro y mi presencia ante ese otro yo, ese modo de darse que posee unas afectaciones intencionales en mí, y cómo me efectúo en la experiencia del otro como ego coexistente, gracias al vinculo y transición constitutiva de las dimensiones pasiva a la activa.

En este caso, Husserl remite la experiencia de manera originaria en una esfera primordial como base de la coexistencia, donde para mí como ego ella es original y la del otro es para mí apresentada, y con el mismo valor monádico es inversamente para el otro desde su experiencia, donde se fundan las percepciones y las apresentaciones como materia y núcleo de las experiencias de coexistencias.

Es esta la razón que fundamenta el argumento que la evaluación como experiencia humana no puede determinarse como un instrumento de medición. Desde la descripción y análisis fenomenológico se podría establecer lo equivocado y erróneo de concebir la evaluación al amparo de la noción de medición o instrumento, porque desde ella se puede establecer que el sujeto no se mide, Husserl define a los sujetos como unidades de desarrollo infinito, como unidades teleológicas, cuya posibilidad de despliegue de desarrollo no depende de dichas unidades, sino de diversos factores asociados a las acciones y no a la teleología que impulsa dicho desarrollo, como se explicó en el parágrafo anterior.

Subsecuentemente, se podría tener que la evaluación como verdadera experiencia humana no es para medir sino para valorar, para reflexionar, como actitud fenomenológica, considerando las transiciones y capas de la pasividad hacia las intencionalidades de la conciencia en esa búsqueda de la razón, como fundamento teleológico humano. En ella hay un yo como campo de trabajo que es infinito $y$ que se constituye en un mundo como correlato de su conciencia cuyo fluir no se agota en la experiencia misma.

Esta relación intersubjetiva es fundamental para considerar la experiencia evaluativa como experiencia humana, toda vez que en ella el 
percibir y darle sentido a la presencia del otro en mí y, luego, el considerar el sentido de mi presencia en el otro como dialéctica constituyente de la subjetividad en la intersubjetividad, fundamente la noción de sujeto inherente a la evaluación educativa desde el terreno abonado desde la antropología fenomenológica en la búsqueda de los fundamentos del conocimiento y la objetividad aplicados al estudio de lo humano como fuente de toda experiencia con la razón como telos que lo dignifica.

Por lo anteriormente descrito, la subjetividad y la intersubjetividad no deben quedar resignadas a una corta dilucidación en la experiencia evaluativa, si son ellas el fundamento constitutivo de la noción de sujeto que le es inherente a dicha experiencia, descrita esta como esencialmente humana y fenomenológica.

\subsection{Instalarse en el mundo, hacia una comprensión del sujeto en la experiencia evaluativa}

Las experiencias de lo humano son dominios que se alimentan de lo inédito y deben romper con la inercia y conformidad con lo dado, estableciéndose una tensión entre cierre y apertura, donde la primera actitud sería la inercia y conformidad del sujeto con lo que se le presenta en su mundo y el segundo movimiento alude a la actitud de constituirse como sujeto y posicionarse en el mundo desde los ámbitos de sentido que forja en aras de trascender la realidad objetual- dada, como subjetividad que dimensiona su experiencia desde su conciencia y en la reflexión permanente de lo que lo constituye. A esto Hugo Zemelman lo denomina actividad y voluntad de pensar como actividad orientada a colocarse ante la realidad- mundo.

En su obra Sujeto: Existencia y Potencia, Hugo Zemelman (1998) se ocupa de la dialéctica de lo mío y lo ajeno desde la posibilidad subjetiva de razonar lo objetivo en su yo interno y el Otro o lo Otro como exterioridad a ese yo. Para él, la estructura reflexiva tiene como núcleo la conciencia del sujeto que reflexiona como voluntad y tarea de primera línea del pensar humano. Por ello, fundamenta e insiste en la necesidad de distanciarnos de lo instrumental o mecanismos que moldean nuestra realidad mediante dominaciones parametrales que prefijan el conocimiento y la razón. El abrirse a lo inédito plantea la posibilidad de una subjetividad que se constituye en el cómo la objetividad se puede afirmar, no sólo "más acá de la medida, sino también más allá de lo medido" (Zemelman, 1998, p. 18).

De tal manera, Zemelman asume que lo fundante de la apertura a la razón y la reflexión desde la conciencia está en el proceso constitutivo de la subjetividad, como escenario para instalarse, posicionarse en la realidad y trascenderla. Lo que propone este posestructuralista latinoamericano es una subjetividad que subordine las verdades 0 lo indeterminado, modos de apropiación creativa y constituyente sobre predicaciones posibles de la realidad, mas no como determinada y fija sin posibilidad de afectación.

Al respecto, Zemelman subraya un error que ha tenido lugar históricamente y que resulta clave en el interés que persigue este estudio de develar la noción de sujeto en la evaluación educativa. Al referirse al proceso inverso que se ha seguido de manera histórica de convertir al objeto en una forma particular de objetividad, determinado por la medición y una lógica de manipulación que no permite extenderse más allá de lo que se prefigura como conocimiento de ese objeto y que subyuga al sujeto que le percibe y aprehende a una objetivación empobrecida, fija y ahistórica porque no le permiten otras modalidades de objetivación distintas a la ya determinada.

Desde estos postulados se reclama una conciencia participativa, un sujeto con el potencial de asomarse por encima de las verdades que le sobrevienen, con el desafío de pensar el potencial del presente histórico sobre la base de reflexionar sobre lo vivido, lo habido 
o lo que no fue y anticipar desde la utopía las profundidades de lo que ha de vivirse o está por venir: "En consecuencia, la racionalidad en la historia consiste y se agota en la potencialidad de lo posible (puede ser y no ser); de ahí su contingencia" (Zemelman, 1998, p. 30).

Zemelman referencia a Edmund Husserl en su obra en los términos de crítica al objetivismo de la ciencia y para utilizar el concepto husserliano de mundo de la vida, en cuanto al esfuerzo del sujeto como vida humana que se apropia de la realidad. Se asume, entonces, un sujeto capaz de problematizar el sentido de su quehacer, en una actitud que se traduzca a la necesidad de realidad por sobre la organización fija de contenidos, "Es lo que configura el perfil de la epistemología del presente potencial, o de la conciencia histórica (Zemelman 2007b 100)".

Por lo tanto, Zemelman entiende la objetivación del sujeto como la construcción de ángulos más abiertos para pensar la realidad con mayor riqueza y la tarea de discernir sobre la opción de razonamiento elegida cuando es confrontada con otra, un sujeto que no se agota en una mono dimensión instaurada, sino que posee múltiples dimensiones de acción como sujeto activo que explora la riqueza de su conciencia como núcleo para reflexionar su mundo y sus experiencias, por lo que se requiere una subjetividad que se considere en su naturaleza constituyente, lo que se corresponde con la exigencia de un sujeto que no sólo sea protagonista, sino el reflejo de que la realidad es histórica y no ahistórica, se constituye y no es prefijada en única dimensión para el sujeto.

Es el reclamo por el sujeto en toda su complejidad concreta, pero también su potenciación como fundamento de su posibilidad de ser constituyente en su realidad, la cual se asume desde aquella que trasciende a la noción de realidad como objeto y que rompe con la homogeneización del pensamiento; esto es, la realidad como "horizonte de construcciones posibles que no reduzca la legitimidad del pensar y del conocimiento al orden, ni el orden a la posibilidad de realización de vida en el plano puramente individual" (p. 57).

La multiplicidad en las dimensiones de acción en el sujeto que reclama Zemelman, alude a una potencialidad de sentidos en esa relación dialéctica del sujeto con el mundo, potencialidad traducida en posibles sentidos de desenvolvimiento y exigencias subjetivas de constitución, operatividad de conciencia sobre lo dado en las experiencias que vive y asume el sujeto, que mediante tal operatividad se coloca ante el mundo, cuya significación de pensarse y actuar inconforme ante lo dado es concebido como incompletud continua, sus movimientos de razón están en constante búsqueda en una "constelación de puntos de activación desde los cuales poder construir un sentido" (p. 69).

De esta manera, se concibe el estar e instalarse en el mundo como condición para pensarlo y desde los ámbitos de sentido encontrados en ese instalarse del sujeto, le permitan comprenderlo, vivirlo de manera consciente, esto es asumir la realidad desde dos planos: como experiencia vivencial y como operatividad de conciencia sobre ámbitos de sentido que constituyen por un lado la subjetividad y por el otro la realidad misma, es decir, la realidad como necesidad de la realidad.

Por consiguiente, instalarse en el mundo dependerá para el sujeto desarrollar la necesidad de realidad como experiencia y como conciencia de apertura. A su vez, Zemelman precisa la necesidad del uso creativo del lenguaje como medio para movilizar el razonamiento constituyente que recurre a los significados para establecer los ámbitos de sentido en cuanto representan modos variados de articulación del sujeto con su mundo, donde se fundamenta y se facilita la interacción con otros, determinándose por lo tanto que tal apertura no es sólo subjetiva sino intersubjetiva. 


\section{CONCLUSIONES EN LA DISCUSIÓN}

Al abordar la subjetividad e intersubjetividad como nociones fenomenológicas al interior de la experiencia evaluativa para interpretar la noción de sujeto o vida humana inherente a ella, se subraya que cada sujeto posee un cuerpo que no viene a estar determinado unívocamente por su estructura biofísica, sino desde sus dos planos corpóreos del yo vivo y el yo vivido que le habitan a esa estructura que hace su entrada en el mundo perceptible del otro y desde donde se delimitan los dos problemas constitutivos a los que Husserl accede de manera explicativa en su obra como parificación y apresentación, conceptos que son fundantes en la intersubjetividad.

Reflexionar, entonces, sobre la experiencia evaluativa abarca los problemas constitutivos que instauran los principios universales de la génesis constitutiva de nosotros como sujetos en correlato con el mundo, en pro de hacer justicia posición contraria a la vivencia ingenua del mundo, desde su constitución sintética pasiva y activa, nos acerca a la problemática de instalarse en el mundo como vía de comprensión del sujeto en la experiencia evaluativa, a partir de lo cual resultan necesarios los aportes de Hugo Zemelman (1998) en su obra Sujeto: Existencia y Potencia, para entender como las experiencias de lo humano son dominios que se alimentan de lo inédito y deben romper con la inercia y conformidad con lo dado, estableciéndose la tensión entre cierre y apertura.

Todo aquello nos exige asumir la experiencia evaluativa no como un episodio donde se mide y se estratifica a un sujeto, sino que remite a una experiencia de vivencias donde el sujeto encuentre el espacio de potenciarse y se constituya en esa interrelación comunicativa y e intersubjetiva con el otro o los otros que coexisten pero que también constituyen y se constituyen en ese mundo de correlaciones de subjetividad e intersubjetividad.

\section{REFERENCIAS BIBLIOGRÁFICAS}

Álvarez, E. (2011). La cuestión del sujeto en la Fenomenología de Husserl. Investigaciones Fenomenológicas(8), 97-149.

Resultó fundamental para los intereses de esta investigación los detalles descriptivos con los que Oswald analiza cómo la dimensión pasiva está en la base del desarrollo constitutivo del mundo, en la medida en que ella es responsable de desplegar todas las estructuras de la subjetividad y por ser el mundo su correlato, no solo se desarrolla la subjetividad, el mundo también, lo cual permite encontrar elementos que se identifican hacia el vector de la intersubjetividad y que resultan claves para entender la evaluación como experiencia humana y por ende antropológica.

Además, desde el pregón en el análisis de Oswald del sujeto que sacude sus hábitos, en aras de desplegarse y levantarse en una
Betancourt-Cadavid, J. (2012). Un Una respuesta al discurso pedagógico de la Modernidad: La pedagogía del caos. Revista En-Clave Social. Corporación Universitaria Lasallista, Antioquia (Colombia). Vol. 1, núm. 1, enero - junio, pp. $42-56$ 
Betancourt-Cadavid, J. (2015) istep:Alternativas antropológicas, históricas $y$ pedagógicas, para la conservación del sujeto en el universo digital. Revista Lasallista de Investigación. Corporación Universitaria Lasallista, Antioquia (Colombia). Vol. 12, núm. 2, pp. 176-185

Bourdieu, P. y Wacquant, L. (2005). Una invitación a la sociología reflexiva. Argentina: Siglo XXI.

Butler, J. (2016). Los sentidos del sujeto. Traducción de Paula Kuffer. Herder Editorial, S.L., Barcelona.

Gysling, J. (2017). La evaluación: ¿dispositivo para promover el aprendizaje de todos o para seleccionar? La formación de profesores en evaluación en Chile. Holanda: Leiden University Repository. Obtenido de http://hdl.handle. net/1887/46245

Husserl, E. (2006). Meditaciones Cartesianas (Tercera ed.). (M. A. Presas, Trad.) Madrid: Tecnos.

López López, A. F. (2016). Entre el sujeto trascendental constituyente $y$ el sujeto constituido y "la paradoja de la subjetividad" descrita por Edmund Husserl. Nuevas Tendencias en Antropología(7), 133-171.

Olave, J. M. (2018). Relatos de experiencias en evaluación. Comprensiones Decoloniales en la escuela Chilena, 2013- 2018. Manizales: Repositorio CINDE. Obtenido de https://repository. cinde.org.co/handle/20.500.11907/2298 ?show=full

Osswald, A. (2016). La fundamentación pasiva de la experiencia. Un estudio sobre la fenomenología de Edmund Husserl. Madrid: Plaza y Valdés.
Runge Peña, A., Hincapié, A., Muñoz, D. y Ospina, C. (2018). El campo disciplinar y profesional de la pedagogía en Colombia. Fondo Editorial Universidad Católica de Oriente.

San Martín, J. (1990). La sexta meditación cartesiana de Eugen Fink. Revista de filosofía(4), 247- 264.

Vargas Bejarano, J. C. (1993). Aproximación al método fenomenológico- genético en: "La crisis de la humanidad europea y la filosofía". Univeristas philosophica(21), 105-123.

Zemelman, H. (1998). Sujeto, existencia y potencia. Barcelona: Anthropos.

Zemelman, H. (2007). El ángel de la historia: determinación y autonomía de la condición humana (Ideas para un programa de humanidades). España: Anthropos Editorial 